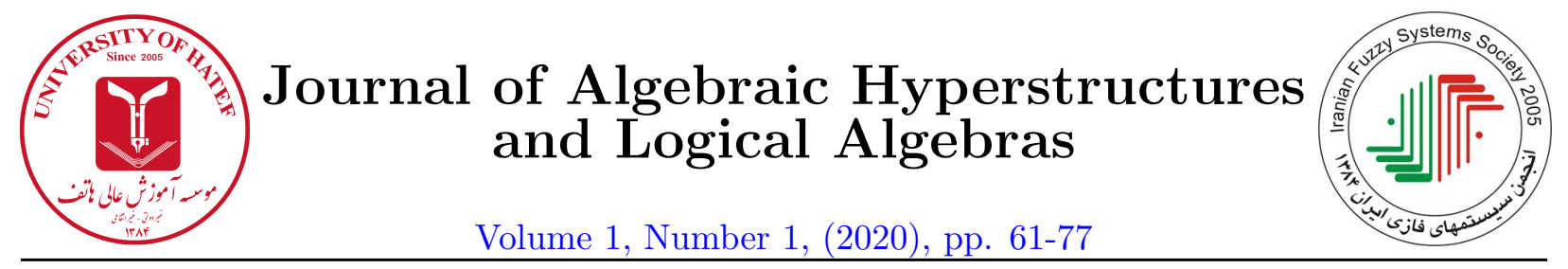

\title{
Results on hoops
}

\author{
R.A. Borzooei ${ }^{1}$ and M. Aaly Kologani ${ }^{2}$ \\ ${ }^{1}$ Department of Mathematics, Shahid Beheshti University, Tehran, Iran \\ ${ }^{2}$ Hatef Higher Education Institute, Zahedan, Iran \\ borzooei@sbu.ac.ir, mona4011@gmail.com
}

\begin{abstract}
In this paper, by considering the notion of hoop, were introduced by Bosbach in [7, 8] under the name of complementary semigroups, we show that there are relations among hoops and some of other logical algebras such as residuated lattices, $M T L$-algebras, $B L$-algebras, $M V$ algebras, $B C K$-algebras, equality algebras, EQ-algebras, $R_{0}$-algebras, Hilbert algebras, Heyting algebras, Hertz algebras, lattice implication algebras and fuzzy implication algebras. The aim of this paper is to find that under what conditions hoops are equivalent to these logical algebras.
\end{abstract}

\section{Article Information}

Corresponding Author:

R.A. Borzooei;

Received: December 2019;

Accepted: Invited paper.

Paper type: Original.

\section{Keywords:}

Hoop, residuated lattice, equality algebra, EQ-algebra, Hilbert algebra.

\section{Introduction}

Hoops were introduced by B. Bosbach in [7, 8] and studied by J.R. Büchi and T.M. Owens and other mathematicians. The study of hoops is motivated by their occurrence both in universal algebra and algebraic logic. Typical examples of hoops include both Brouwerian semilattices and the positive cones of lattice ordered Abelian groups, while hoops structurally enriched with normal multiplicative operators naturally generalize the normal Boolean algebras with operators. In the last years, hoops theory was enriched with deep structure theorems[1, 2, 3, 5, 6]. Many of these results have a strong impact with fuzzy logic. Particularly, from the structure theorem of finite basic hoops one obtains an elegant short proof of the completeness theorem for propositional basic logic introduced by Hájek. The algebraic structures corresponding to Hájek's propositional (fuzzy) basic logic, BL-algebras, are particular cases of hoops. The main example of BL-algebra is the interval $[0,1]$ endowed with the structure induced by a t-norm. MV-algebras, product algebras and Gödel algebras are the most known classes of BL-algebras. Now, in this paper we study

https://doi.org/10.29252/hatef.jahla.1.1.5 
the relation between hoops and other logical algebras such as a residuated lattice, $M T L$-algebra, $B L$-algebra, $M V$-algebra, $B C K$-algebra, Hilbert algebra, etc.

\section{Preliminaries}

Bellow we recall some definitions that will be used in the rest of the paper.

Definition 2.1. [1] $A$ hoop is an algebra $(A, \odot, \rightarrow, 1)$ of type $(2,2,0)$ such that, for all $x, y, z \in A$ : $(H P 1)(A, \odot, 1)$ is a commutative monoid,

$(H P 2) \quad x \rightarrow x=1$,

$(H P 3) \quad(x \odot y) \rightarrow z=x \rightarrow(y \rightarrow z)$,

$(H P 4) \quad x \odot(x \rightarrow y)=y \odot(y \rightarrow x)$.

$A$ relation $\leq$ on hoop $A$ is defined by $x \leq y$ if and only if $x \rightarrow y=1$, then $\leq$ is a partial order relation on $A$. A hoop $A$ is called bounded if there is an element $0 \in A$ such that $0 \leq x$, for all $x \in A$. Let $A$ be a bounded hoop. The negation" ' " on $A$ is defined by, $x^{\prime}=x \rightarrow 0$, for all $x \in A$. If $\left(x^{\prime}\right)^{\prime}=x$, for all $x \in A$, then the bounded hoop $A$ is said to have the double negation property, or (DNP) for short. Finally, for any $n \in \mathbb{N}$, defined $x^{0}=1$ and $x^{n}=x^{n-1} \odot x$. (See [1, 7])

The following proposition provides some properties of hoops.

Proposition 2.2. [7, 8] Let $A$ be a hoop. Then, for all $x, y, z \in A$, the following properties hold:

(i) $(A, \leq)$ is a $\wedge$-semilattice with $x \wedge y=x \odot(x \rightarrow y)$;

(ii) $x \odot y \leq z$ if and only if $x \leq y \rightarrow z$;

(iii) $x \odot y \leq x, y$;

(iv) $\quad x \leq y \rightarrow x$;

(v) $1 \rightarrow x=x$

(vi) $\quad x \rightarrow 1=1$;

(vii) $y \leq(y \rightarrow x) \rightarrow x$;

(viii) $\quad x \leq(x \rightarrow y) \rightarrow x$

(ix) $\quad(x \rightarrow y) \leq(y \rightarrow z) \rightarrow(x \rightarrow z)$;

(x) $\quad(x \rightarrow y) \odot(y \rightarrow z) \leq(x \rightarrow z)$;

(xi) $\quad x \leq y$ implies $x \odot z \leq y \odot z$;

(xii) $\quad x \leq y$ implies $z \rightarrow x \leq z \rightarrow y$;

(xiii) $x \leq y$ implies $y \rightarrow z \leq x \rightarrow z$;

(xiv) $\quad x \rightarrow(y \wedge z)=(x \rightarrow y) \wedge(x \rightarrow z)$.

Proposition 2.3. 15] Let $A$ be a hoop and for any $x, y \in A$,

$$
x \vee y=((x \rightarrow y) \rightarrow y) \wedge((y \rightarrow x) \rightarrow x) .
$$

Then the following conditions are equivalent:

(i) $\vee$ is associative;

(ii) $\quad x \leq y$ implies $x \vee z \leq y \vee z$, for all $x, y, z \in A$;

(iii) $\quad x \vee(y \wedge z) \leq(x \vee y) \wedge(x \vee z)$, for all $x, y, z \in A$;

(iv) $\vee$ is the join operation on $A$.

Definition 2.4. A hoop $A$ is called a $\vee$-hoop, if it satisfies in the one of equivalent conditions of Proposition 2.3. 
Proposition 2.5. 15] Let $A$ be $a \vee$-hoop. Then for all $x, y, z \in A$,

$$
(x \vee y) \rightarrow z=(x \rightarrow z) \wedge(y \rightarrow z) .
$$

Remark 2.6. [15] $\vee$-hoop $(A, \vee, \wedge)$ is a distributive lattice.

\section{Relations among hoops and some logical algebras}

In this section, we investigate the relations among hoops and some logical algebras such as residuated lattices, $M T L$-algebras, $B L$-algebras, $M V$-algebras, $B C K$-algebras, $R_{0}$-algebras, Hilbert algebras, Heyting algebras, Hertz algebras and fuzzy implication algebras.

\subsection{Relation with residuated lattices ( $M T L, B L$ and $M V$-algebras)}

Definition 3.1. 22] A residuated lattice is an algebra $(L, \vee, \wedge, \odot, \rightarrow, 0,1)$ of type $(2,2,2,2,0,0)$ satisfying the following axioms:

$(R L 1) \quad(L, \vee, \wedge, 0,1)$ is a bounded lattice, $(R L 2) \quad(L, \odot, 1)$ is a commutative monoid,

$(R L 3) \quad x \odot y \leq z$ if and only if $x \leq y \rightarrow z$, for all $x, y, z \in L$.

Proposition 3.2. [20] Let $L$ be a residuated lattice. Then, for any $x, y, z \in L$, the following conditions are equivalent:

$\left(r_{1}\right) \quad x \rightarrow(y \vee z)=(x \rightarrow y) \vee(x \rightarrow z)$,

$\left(r_{2}\right) \quad(x \wedge y) \rightarrow z=(x \rightarrow z) \vee(y \rightarrow z)$,

$\left(r_{3}\right) \quad(x \rightarrow y) \vee(y \rightarrow x)=1$.

Definition 3.3. [11, 14, 16] Let $L$ be a residuated lattice. Then $L$ is called;

(i) a divisible residuated lattice, if for any $x, y \in L$,

$$
x \wedge y=x \odot(x \rightarrow y),
$$

(ii) an MTL-algebra, if for any $x, y \in L$,

$$
(x \rightarrow y) \vee(y \rightarrow x)=1, \quad(M 1)
$$

(iii) a BL-algebra, if $L$ is an MTL-algebra and, for any $x, y \in L$,

$$
x \wedge y=x \odot(x \rightarrow y), \quad(B 1)
$$

(iv) an $M V$-algebra, if $L$ is a $B L$-algebra and, for any $x \in L$,

$$
x^{\prime \prime}=x, \quad(D N P)
$$

where $x^{\prime}=x \rightarrow 0$.

Proposition 3.4. 3] Let $L$ be a divisible residuated lattice. Then $L$ is a bounded hoop which is a $\vee$-semilattice.

Theorem 3.5. $(A, \odot, \rightarrow, 0,1)$ is a bounded $\vee$-hoop if and only if $(A, \wedge, \vee, \odot, \rightarrow, 0,1)$ is a divisible residuated lattice. 
Proof. $(\Rightarrow)$ Let $(A, \odot, \rightarrow, 0,1)$ be a bounded $\vee$-hoop. It is clear that $(A, \odot, 1)$ is a commutative monoid. Moreover, by Remark 2.6, $(A, \wedge, \vee, 0,1)$ is a bounded distributive lattice. Now, let $x \odot y \leq z$, for some $x, y, z \in A$. Then $(x \odot y) \rightarrow z=1$, and so by (HP3), $x \rightarrow(y \rightarrow z)=1$. Thus, $x \leq y \rightarrow z$. By the similar way, we can prove the other side. Hence, $A$ is a residuated lattice. Also, by Proposition 2.2(i), for any $x, y \in A, x \wedge y=x \odot(x \rightarrow y)$. Therefore, $(A, \wedge, \vee, \odot, \rightarrow, 0,1)$ is a divisible residuated lattice.

$(\Leftarrow)$ By Proposition 3.4 , the proof is clear.

Let us remark that residuated lattices and hoops are incomparable. Indeed, not all hoops are residuated lattices. It is noticeable that, hoop is a meet semilattice with respect to the meet operator $a \wedge b=a \odot(a \rightarrow b)$ but it has not a lattice structure. The following counterexample indicates that any residuated lattices is not a hoop, in general.

Example 3.6. Let $A=[0,1]$. Then, for all $x, y \in A$, define the operations $\wedge, \vee, \odot$ and $\rightarrow$ on $A$ as follows:

$$
\begin{gathered}
x \wedge y=\min \{x, y\} \quad \text { and } \quad x \vee y=\max \{x, y\}, \\
x \odot y=\left\{\begin{array}{ccc}
0 \quad, \quad x+y \leq 1 \\
x \wedge y \quad, \quad x+y>1
\end{array} \quad \text { and } \quad x \rightarrow y=\left\{\begin{array}{cc}
1 & , x \leq y \\
(1-x) \vee x \quad, & x>y
\end{array}\right.\right.
\end{gathered}
$$

Then $(A, \vee, \wedge, \odot, \rightarrow, 0,1)$ is a residuated lattice. Now, let $x>y$, for some $x, y \in A$. Then $x \odot(x \rightarrow y)=x \odot((1-x) \vee x)$. If $(1-x) \vee x=1-x$, since $x+(1-x)=1$, we have $x \odot(1-x)=0 \neq y=x \wedge y$. If $(1-x) \vee x=x$, then $x \odot x=0$ or $x$, and so $x \wedge y=y \neq x \odot(x \rightarrow y)$. Therefore, $A$ is not a hoop.

Theorem 3.7. $(A, \odot, \rightarrow, 0,1)$ is a bounded $\vee$-hoop with condition $(M 1)$ if and only if $(A, \wedge, \vee, \odot, \rightarrow$ $, 0,1)$ is an $M T L$-algebra with condition (B1).

Proof. $(\Rightarrow)$ Let $(A, \odot, \rightarrow, 0,1)$ be a bounded $\vee$-hoop with condition (M1). By Theorem 3.5. $(A, \wedge, \vee, \odot, \rightarrow, 0,1)$ is a divisible residuated lattice. So, we have (B1). Since $A$ is a residuated lattice and has condition (M1), by Defenition 3.3(ii), $(A, \wedge, \vee, \odot, \rightarrow, 0,1)$ is an $M T L$-algebra.

$(\Leftarrow)$ Since $(A, \wedge, \vee, \odot, \rightarrow, 0,1)$ is an $M T L$-algebra, $A$ is a residuated lattice with condition (M1). Moreover, since $A$ has condition (B1), $A$ is a divisible residuated lattice. Hence, by Theorem 3.5 . $(A, \odot, \rightarrow, 0,1)$ is a bounded $\vee$-hoop. Therefore, $(A, \odot, \rightarrow, 0,1)$ is a bounded $\vee$-hoop with condition (M1).

Corollary 3.8. $(A, \wedge, \vee, \odot, \rightarrow, 0,1)$ is a BL-algebra if and only if $(A, \odot, \rightarrow, 0,1)$ is a bounded $\checkmark$-hoop with condition (M1).

Proof. According to Definition 3.3(iii), every $M T L$-algebra with condition (B1) is a $B L$-algebra, then by Theorem 3.7, the proof is clear.

Theorem 3.9. $(A, \odot, \rightarrow, 0,1)$ is a bounded $\vee$-hoop with (DNP) and condition (M1) if and only if $\left(A, \odot, \rightarrow,{ }^{\prime}, 1\right)$ is an $M V$-algebra. 
Proof. $(\Rightarrow)$ Let $(A, \odot, \rightarrow, 0,1)$ be a bounded $\vee$-hoop with condition (M1). Then by Corollary 3.8 , $(A, \wedge, \vee, \odot, \rightarrow, 0,1)$ is a $B L$-algebra. Since $A$ has $(\mathrm{DNP})$, by Definition 3.3 (iv), $\left(A, \odot, \rightarrow,^{\prime}, 1\right)$ is an $M V$-algebra.

$(\Leftarrow)$ Let $\left(A, \odot, \rightarrow,^{\prime}, 1\right)$ be an $M V$-algebra. Since any $M V$-algebra is a $B L$-algebra with (DNP), by Corollary 3.8, $(A, \odot, \rightarrow, 0,1)$ is a bounded $\vee$-hoop with (DNP) and condition (M1).

Example 3.10. Let $A=\{0, a, b, 1\}$ be a poset such that $0 \leq a, b \leq 1$. Define the operations $\rightarrow$ and $\odot$ on $A$ as follows,

\begin{tabular}{c|cccc}
$\rightarrow$ & 0 & $a$ & $b$ & 1 \\
\hline 0 & 1 & 1 & 1 & 1 \\
$a$ & $b$ & 1 & $b$ & 1 \\
$b$ & $a$ & $a$ & 1 & 1 \\
1 & 0 & $a$ & $b$ & 1
\end{tabular}

\begin{tabular}{c|cccc}
$\odot$ & 0 & $a$ & $b$ & 1 \\
\hline 0 & 0 & 0 & 0 & 0 \\
$a$ & 0 & $a$ & 0 & $a$ \\
$b$ & 0 & 0 & $b$ & $b$ \\
1 & 0 & $a$ & $b$ & 1
\end{tabular}

Then $(A, \odot, \rightarrow, 0,1)$ is a $\vee$-hoop with (DNP) and condition (M1).

Proposition 3.11. $\left(A, \odot, \rightarrow,{ }^{\prime}, 1\right)$ is an $M V$-algebra if and only if there exists an algebraic structure $(A, \oplus, \neg, 0)$ of type $(2,1,0)$, where $(A, \oplus, 0)$ is a commutative monoid with neutral element 0 and, for all $x, y \in A$ :

$\left(M V_{1}\right) \neg(\neg x)=x$,

$\left(M V_{2}\right) \quad x \oplus 1=1$, where $1=\neg 0$,

$\left(M V_{3}\right) \quad x \oplus \neg(x \oplus \neg y)=y \oplus \neg(y \oplus \neg x)$.

Proof. $(\Rightarrow)$ Suppose $\left(A, \odot, \rightarrow,^{\prime}, 1\right)$ is an $M V$-algebra. Define $x \oplus y=x^{\prime} \rightarrow y$ and $\neg x=x^{\prime}$, for all $x, y \in A$. It is easy to see that $(A, \oplus, 0)$ is a commutative monoid that satisfying in conditions $\left(M V_{1}\right),\left(M V_{2}\right)$ and $\left(M V_{3}\right)$.

$(\Leftarrow)$ Let $(A, \oplus, 0)$ be a commutative monoid with constant element 0 and satisfying the conditions $\left(M V_{1}\right),\left(M V_{2}\right)$ and $\left(M V_{3}\right)$. For all $x, y \in A$, we define,

$$
x \odot y=\neg(\neg x \oplus \neg y), x \rightarrow y=\neg x \oplus y, x^{\prime}=\neg x, x \wedge y=(x \oplus \neg y) \odot y \text { and } x \vee y=x \odot \neg y
$$

Then $\left(A, \odot, \rightarrow,^{\prime}, 1\right)$ is an $M V$-algebra.

Theorem 3.12. If $(A, \odot, \rightarrow, 0,1)$ is a bounded hoop with $(D N P)$, then $(A, \oplus, 0)$ is an $M V$-algebra.

Proof. Suppose $(A, \odot, \rightarrow, 0,1)$ is a bounded hoop with (DNP). It is enough to prove that the conditions of Proposition 3.11 hold. Define $x \oplus y=\left(x^{\prime} \odot y^{\prime}\right)^{\prime}$, for all $x, y \in A$. Routine calculation shows that $(A, \oplus, 0)$ is a commutative monoid. Since $A$ has (DNP), we have $\left(M V_{1}\right)$. Moreover, $x \oplus 1=\left(x^{\prime} \odot 0\right)^{\prime}=1$, so we have $\left(M V_{2}\right)$. Also, by definition of $\oplus$ and (DNP), we have $x \oplus\left(x \oplus y^{\prime}\right)^{\prime}=$ $\left(x^{\prime} \odot\left(x^{\prime} \odot y\right)^{\prime}\right)^{\prime}$. By $(\mathrm{HP} 3)$,

$$
\left(x^{\prime} \odot\left(x^{\prime} \odot y\right)^{\prime}\right)^{\prime}=\left(x^{\prime} \odot\left(x^{\prime} \rightarrow y^{\prime}\right)\right)^{\prime} .
$$

Then by (HP4),

$$
x \oplus\left(x \oplus y^{\prime}\right)^{\prime}=\left(y^{\prime} \odot\left(y^{\prime} \rightarrow x^{\prime}\right)\right)^{\prime}=y \oplus\left(y \oplus x^{\prime}\right)^{\prime} .
$$

Hence, $\left(M V_{3}\right)$ holds. Therefore, by Proposition 3.11 $(A, \oplus, 0)$ is an $M V$-algebra.

Corollary 3.13. $(A, \oplus, 0)$ is an $M V$-algebra if and only if $(A, \odot, \rightarrow, 0,1)$ is a bounded $\vee$-hoop with $(D N P)$.

Proof. By Theorems 3.12 and 3.9 , the proof is clear. 


\subsection{Relation with $B C K(B C I)$-algebras}

In this section, we investigate the relation among hoop and $B C K$ and $B C I$-algebras.

Definition 3.14. [18] $A$ BCI-algebra is an algebra $(A, *, 0)$ of type $(2,0)$ that, for any $x, y, z \in A$, it satisfies the following conditions:

$(B C I 1) \quad((x * y) *(x * z)) *(z * y)=0$,

$(B C I 2) \quad(x *(x * y)) * y=0$,

$(B C I 3) \quad x * x=0$,

$(B C I 4) \quad x * y=0$ and $y * x=0$ imply $x=y$.

Relation " $\leq$ " on BCI-algebra $A$ which is defined by $x \leq y$ if and only if $x * y=0$, for any $x, y \in A$, is a partial order. If a BCI-algebra $A$ satisfies $0 * x=0$, for any $x \in A$, then $A$ is called a BCK-algebra. A BCK-algebra $A$ is called non-trivial if $A \neq\{0\}$. If $A$ is a $B C K$-algebra and the poset $(A, \leq)$ is a lattice, then $A$ is called a $B C K$-lattice. $A B C K$-lattice $(A, \leq, *, 0)$ is denoted by $(A, \wedge, \vee, *, 0)$, where $x \leq y$ if and only if $x \wedge y=x$ if and only if $x \vee y=y$, for all $x, y \in A$. A $B C K$-algebra $(A, \leq, *, 0)$ is called a $B C K$-algebra with product $(B C K(P)$-algebra), if, for any $x, y \in A$, it is satisfying in the condition $(P), x \odot y=\min \{z \mid x \leq y \rightarrow z\}$ exists. $A$ BCK-algebra $A$ is called implicative if $x *(y * x)=x$ and is commutative if $x *(x * y)=y *(y * x)$, for any $x, y \in A$. (See [17, 18])

Theorem 3.15. [12] Every hoop is a BCK-algebra.

Corollary 3.16. Every hoop is a BCI-algebra.

Proof. By Definition 3.14 and Theorem 3.15, the proof is clear.

Lemma 3.17. [17] Let $(A, \leq, *, 0)$ be a $B C K(P)$-algebra. Then,

(i) $(A, \odot, 0)$ is a commutative monoid with the greatest element 0 ,

(ii) if $A$ is a $B C K(P)$-lattice such that $x^{2}=x$, for any $x \in A$, then $x \wedge y=x \odot(x \rightarrow y)$, for any $x, y \in A$.

(iii) $(z * y) * x=z *(x \odot y)$, for any $x, y, z \in A$.

Theorem 3.18. Let $(A, \leq, *, 0)$ be a $B C K(P)$-lattice. If $x^{2}=x$, for any $x \in A$, then $(A, \odot, \rightarrow, 1)$ is a hoop.

Proof. Let $A$ be a $B C K(P)$-lattice. Define $y \rightarrow x=x * y$, for any $x, y \in A$. Since $A$ is a $\mathrm{BCK}(\mathrm{P})$ lattice, by Lemma 3.17(i), $(A, \odot, 1)$ is a commutative monoid, (HP1) holds. Since, $x^{2}=x$, for any $x \in A$, by Lemma 3.17(ii), $x \wedge y=x \odot(x \rightarrow y)$, and so (HP4) holds. Moreover, since $A$ is a $B C K$-algebra, we have $x * x=0$, for any $x \in A$. Since $1=x \rightarrow x=x * x=0$, we have (HP2). Also, by Lemma 3.17 (iii), $(z * y) * x=z *(x \odot y)$, for any $x, y, z \in A$, and so $x \rightarrow(y \rightarrow z)=(x \odot y) \rightarrow z$. Therefore, $(A, \odot, \rightarrow, 1)$ is a hoop.

Example 3.19. Let $A=\{0, a, b, 1\}$ be a set such that $0 \leq a \leq b \leq 1$. Define the operation $\rightarrow$ on $A$ as follows,

\begin{tabular}{c|cccc}
$\rightarrow$ & 0 & $a$ & $b$ & 1 \\
\hline 0 & 1 & 1 & 1 & 1 \\
$a$ & 0 & 1 & 1 & 1 \\
$b$ & 0 & $a$ & 1 & 1 \\
1 & 0 & $a$ & $b$ & 1
\end{tabular}


Then $(A, \rightarrow, 0,1)$ is a bounded BCK-algebra. Since $A$ is a chain, it is easy to see that $A$ is a $B C K$-lattice. The operation $\odot$ on $A$ is given by the following table:

\begin{tabular}{c|cccc}
$\odot$ & 0 & $a$ & $b$ & 1 \\
\hline 0 & 0 & 0 & 0 & 0 \\
$a$ & 0 & $a$ & $a$ & $a$ \\
$b$ & 0 & $a$ & $b$ & $b$ \\
1 & 0 & $a$ & $b$ & 1
\end{tabular}

Thus, $A$ is a $B C K(P)$-lattice such that, for any $x \in A, x^{2}=x$.

Next, we investigate the relation among hoop and commutative and implicative BCK-algebra.

Lemma 3.20. If $(A, \odot, \rightarrow, 0,1)$ is a bounded $\vee$-hoop with $(D N P)$, then for any $x, y \in A$,

$$
x \rightarrow y=y^{\prime} \rightarrow x^{\prime} \quad, \quad(x \rightarrow y) \rightarrow y=(y \rightarrow x) \rightarrow x .
$$

Proof. Let $(A, \odot, \rightarrow, 0,1)$ be a bounded $\vee$-hoop with (DNP) and $x, y \in A$. Since $y^{\prime \prime}=y$, we have $x \rightarrow y=x \rightarrow y^{\prime \prime}$. Then by (HP3),

$$
x \rightarrow y^{\prime \prime}=x \rightarrow\left(y^{\prime} \rightarrow 0\right)=y^{\prime} \rightarrow(x \rightarrow 0),
$$

and so $x \rightarrow y=y^{\prime} \rightarrow x^{\prime}$. Now, we prove that $(x \rightarrow y) \rightarrow y=(y \rightarrow x) \rightarrow x$. Since $y^{\prime \prime}=y$ and $x \rightarrow y=y^{\prime} \rightarrow x^{\prime}$, we have $(x \rightarrow y) \rightarrow y=\left(y^{\prime} \rightarrow x^{\prime}\right) \rightarrow y^{\prime \prime}$. Thus, by (HP3) and Proposition 2.2(i), we obtain that

$$
(x \rightarrow y) \rightarrow y=\left(y^{\prime} \rightarrow x^{\prime}\right) \rightarrow y^{\prime \prime}=\left(y^{\prime} \rightarrow x^{\prime}\right) \rightarrow\left(y^{\prime} \rightarrow 0\right)=\left(y^{\prime} \odot\left(y^{\prime} \rightarrow x^{\prime}\right)\right) \rightarrow 0=\left(y^{\prime} \wedge x^{\prime}\right)^{\prime},
$$

By the similar way, we can prove that $(y \rightarrow x) \rightarrow x=\left(y^{\prime} \wedge x^{\prime}\right)^{\prime}$. Hence, $(x \rightarrow y) \rightarrow y=(y \rightarrow$ $x) \rightarrow x$.

Theorem 3.21. If $(A, \odot, \rightarrow, 0,1)$ is a bounded $\vee$-hoop with $(D N P)$, then $(A, *, 0)$ is a commutative $B C K$-algebra.

Proof. Let $(A, \odot, \rightarrow, 0,1)$ be a bounded $\vee$-hoop with (DNP). Then by Theorem $3.15, A$ is a $B C K$ algebra. Define $x * y=y \rightarrow x$, for any $x, y \in A$ and consider that the constant is 1 . Then by definition of $*, x *(x * y)=(y \rightarrow x) \rightarrow x$, and so by Lemma 3.20 ,

$$
x *(x * y)=(y \rightarrow x) \rightarrow x=(x \rightarrow y) \rightarrow y=y *(y * x)
$$

Therefore, $(A, *, 0)$ is a commutative $B C K$-algebra.

The following example shows that every bounded $\vee$-hoop is not a commutative $B C K$-algebra, in general.

Example 3.22. Let $A=\{0, a, b, c, 1\}$ be a set such that $0 \leq c \leq a, b \leq 1$, but $a$ and $b$ are incomparable. Define the operations $\wedge, \vee, \odot$ and $\rightarrow$ on $A$ as follows:

\begin{tabular}{l|lllll}
$\rightarrow$ & 0 & $c$ & $a$ & $b$ & 1 \\
\hline 0 & 1 & 1 & 1 & 1 & 1 \\
$c$ & 0 & 1 & 1 & 1 & 1 \\
$a$ & 0 & $b$ & 1 & $b$ & 1 \\
$b$ & 0 & $a$ & $a$ & 1 & 1 \\
1 & 0 & $c$ & $a$ & $b$ & 1
\end{tabular}

\begin{tabular}{c|ccccc}
$\odot$ & 0 & $c$ & $a$ & $b$ & 1 \\
\hline 0 & 0 & 0 & 0 & 0 & 0 \\
$c$ & 0 & $c$ & $c$ & $c$ & $c$ \\
$a$ & 0 & $c$ & $a$ & $c$ & $a$ \\
$b$ & 0 & $c$ & $c$ & $b$ & $b$ \\
1 & 0 & $c$ & $a$ & $b$ & 1
\end{tabular}




\begin{tabular}{l|lllll}
$\vee$ & 0 & $c$ & $a$ & $b$ & 1 \\
\hline 0 & 0 & $c$ & $a$ & $b$ & 1 \\
$c$ & $c$ & $c$ & $a$ & $b$ & 1 \\
$a$ & $a$ & $a$ & $a$ & 1 & 1 \\
$b$ & $b$ & $b$ & 1 & $b$ & 1 \\
1 & 1 & 1 & 1 & 1 & 1
\end{tabular}

\begin{tabular}{c|ccccc}
$\wedge$ & 0 & $c$ & $a$ & $b$ & 1 \\
\hline 0 & 0 & 0 & 0 & 0 & 0 \\
$c$ & 0 & $c$ & $c$ & $c$ & $c$ \\
$a$ & 0 & $c$ & $a$ & $c$ & $a$ \\
$b$ & 0 & $c$ & $c$ & $b$ & $b$ \\
1 & 0 & $c$ & $a$ & $b$ & 1
\end{tabular}

Then $(A, \odot, \rightarrow, 0,1)$ is a bounded $\vee$-hoop. Since $(c \rightarrow 0) \rightarrow 0=1 \neq c, A$ does not have (DNP). Moreover, by Theorem 3.15, A is a BCK-algebra, but it is not a commutative ones. Because,

$$
0 *(0 * a)=(a \rightarrow 0) \rightarrow 0=1 \neq a=(0 \rightarrow a) \rightarrow a=a *(a * 0) .
$$

Proposition 3.23. Let $(A, \odot, \rightarrow, 0,1)$ be a bounded $\vee$-hoop with $(D N P)$ such that $x^{2}=x$, for any $x \in A$. Then $(A, *, 0)$ is an implicative $B C K$-algebra.

Proof. Let $(A, \odot, \rightarrow, 0,1)$ be a bounded $\vee$-hoop with (DNP) such that $x^{2}=x$, for any $x \in A$. Define $x * y=y \rightarrow x$, for any $x, y \in A$ and consider that the constant is 1 . By Theorem 3.21. $(A, *, 0)$ is a commutative BCK-algebra. Hence, $x *(x * y)=y *(y * x)$, for any $x, y \in A$. By Proposition 2.2(viii), $x \leq(x \rightarrow y) \rightarrow x$, for any $x, y \in A$, and so $x *(y * x) \leq x$. Since $A$ has (DNP) and $x^{2}=x$, for any $x \in A$, we have,

$$
x *(x *(y * x))=(y * x) *((y * x) * x)=(y * x) *\left(y * x^{2}\right)=(y * x) *(y * x)=0 .
$$

Then $x \leq x *(y * x)$, and so $x *(y * x)=x$. Therefore, $(A, *, 0)$ is an implicative $B C K$-algebra.

Example 3.24. (i) Let $A$ be the hoop as in Example 3.10, then $A$ is a $\vee$-hoop with (DNP) and for all $x \in A, x^{2}=x$.

(ii) Let $A$ be a hoop as in Example 3.22, then $A$ is a BCK-algebra, but is not an implicative $B C K$-algebra, because,

$$
a *(0 * a)=(a \rightarrow 0) \rightarrow a=1 \neq a .
$$

Corollary 3.25. Let $(A, *, 0)$ be a BCI-algebra. If, for any $x, y \in A, x \rightarrow y=y * x$, then $(A, \odot, \rightarrow, 1)$ is a hoop, if it satisfies in the following conditions:

(i) $0 * x=0$, for any $x \in A$;

(ii) $(A, \leq)$ is a lattice;

(iii) $x \odot y=\min \{z \mid x \leq y \rightarrow z\}$ exists, for any $x, y \in A$;

(iv) if $x^{2}=x$, then $x \wedge y=x \odot(x \rightarrow y)$, for any $x, y \in A$.

Proof. Let $(A, *, 0)$ be a $B C I$-algebra. By (i) and (iii), $(A, \leq, *, 0)$ is a $B C K(P)$-algebra. Then by Lemma 3.17 and Theorem 3.18 , the proof is clear.

\subsection{Relation with equality and EQ-algebras}

In this section, we study the relation among hoops, equality algebras and EQ-algebras.

Definition 3.26. [19] An algebraic structure $(E, \wedge, \sim, 1)$ of type $(2,2,0)$ is called an equality algebra if for any $x, y, z \in E$ the following statements hold:

$(E 1)(E, \wedge, 1)$ is a commutative idempotent monoid(i.e. meet semilattice with top element 1$)$.

(E2) $x \sim y=y \sim x$.

(E3) $x \sim x=1$. 
(E4) $x \sim 1=x$.

(E5) $x \leq y \leq z$ implies $x \sim z \leq y \sim z$ and $x \sim z \leq x \sim y$.

$(E 6) x \sim y \leq(x \wedge z) \sim(y \wedge z)$.

$(E 7) x \sim y \leq(x \sim z) \sim(y \sim z)$.

The operation $\wedge$ is called meet(infimum) and $\sim$ is an equality operation. We write $x \leq y$ if and only if $x \wedge y=x$, as usual. Define the following two derived operations, the implication and the equivalence operation of the equality algebra by,

$$
x \rightarrow y=x \sim(x \wedge y) \text { and } x \leftrightarrow y=(x \rightarrow y) \wedge(y \rightarrow x)
$$

Theorem 3.27. [19, 25] The following two statements hold:

(i) For any equality algebra $\mathcal{E}=(E, \wedge, \sim, 1), \psi(\mathcal{E})=(E, \wedge, \rightarrow, 1)$ is a BCK-meet-semilattice, where $x \rightarrow y=(x \wedge y) \sim x$, for any $x, y \in E$.

(ii) For any $B C K$-meet-semilattice $\mathcal{B}=(E, \wedge, \rightarrow, 1)$ such that, for any $x, y, z \in E, x \rightarrow(y \wedge z)=$ $(x \rightarrow y) \wedge(x \rightarrow z)$, we get that $\varphi(\mathcal{B})=(E, \wedge, \leftrightarrow, 1)$ is an equality algebra, where $x \leftrightarrow y=(x \rightarrow$ $y) \wedge(y \rightarrow x)$. Moreover, the implication of $\varphi(\mathcal{B})$ coincides with $\rightarrow$, that is $x \rightarrow y=x \leftrightarrow(x \wedge y)$.

Theorem 3.28. 25] For any hoop $\mathcal{A}=(A, \odot, \rightarrow, 1)$, the structure $\psi(\mathcal{A})=(A, \wedge, \leftrightarrow, 1)$ is an equality algebra, where $x \leftrightarrow y=(x \rightarrow y) \wedge(y \rightarrow x)$, for $x, y \in A$. Moreover, the implication of $\psi(\mathcal{A})$ coincides with $\rightarrow$, that is $x \rightarrow y=x \leftrightarrow(x \wedge y)$.

Theorem 3.29. [25] Let $(E, \wedge, \sim, 1)$ be an involutive equality algebra and $x \odot y=\left(x \rightarrow y^{\prime}\right)^{\prime}$, for any $x, y \in E$. If $x \wedge y=x \odot(x \rightarrow y)$, then $(E, \odot, \rightarrow, 1)$ is a hoop.

Theorem 3.30. 25] Let $(E, \wedge, \sim, 1)$ be an equality algebra that is not involutive and $x \odot y=x \wedge y$, for any $x, y \in E$. If $(x \odot y) \rightarrow z=x \rightarrow(y \rightarrow z)$, then $(E, \odot, \rightarrow, 1)$ is a hoop.

Definition 3.31. 13] An algebraic structure $(E, \wedge, \otimes, \sim, 1)$ of type $(2,2,2,0)$ is called an EQalgebra if for any $x, y, z, w \in E$ the following statements hold:

(EQ1) $(E, \wedge, 1)$ is a commutative idempotent monoid(i.e. meet semilattice with top element 1).

$(E Q 2)(E, \otimes, 1)$ is a monoid such that the operation $\otimes$ is isotone.

$(E Q 3) x \sim x=1$.

$(E Q 4)((x \wedge y) \sim z) \otimes(w \sim x) \leq z \sim(w \wedge y)$.

$(E Q 5)(x \sim y) \otimes(z \sim w) \leq(x \sim z) \sim(y \sim w)$.

$(E Q 6)(x \wedge y \wedge z) \sim x \leq(x \wedge y) \sim x$.

$(E Q 7) x \otimes y \leq x \sim y$.

The operations $\wedge, \otimes$, and $\sim$ are called meet, multiplication, and fuzzy equality, respectively. For any $a, b \in E$, we set $a \leq b$ if and only if $a \wedge b=a$ and we defined the binary operation implication on $E$ by, $a \rightarrow b=(a \wedge b) \sim a$. Also, in particular $1 \rightarrow a=1 \sim a=\tilde{a}$. If $E$ contains a bottom element 0 , we denote it by BEQ-algebra and then an unary operation $\neg$ is defined on $E$ by $\neg a=a \sim 0$.

An EQ-algebra is good if $x \sim 1=x$.

Theorem 3.32. 25] Every good EQ-algebra $(E, \wedge, \otimes, \sim, 1)$ is an equality algebra.

Corollary 3.33. For any hoop $\mathcal{A}=(A, \odot, \rightarrow, 1)$, the structure $\psi(\mathcal{A})=(A, \wedge, \leftrightarrow, 1)$ is an $E Q$ algebra, where $x \leftrightarrow y=(x \rightarrow y) \wedge(y \rightarrow x)$ and $x \sim 1=x$, for $x, y \in A$. Moreover, the implication of $\psi(\mathcal{A})$ coincides with $\rightarrow$, that is $x \rightarrow y=x \leftrightarrow(x \wedge y)$.

Corollary 3.34. Let $(E, \wedge, \sim, 1)$ be an involutive good EQ-algebra and $x \odot y=\left(x \rightarrow y^{\prime}\right)^{\prime}$, for any $x, y \in E$. If $x \wedge y=x \odot(x \rightarrow y)$, then $(E, \odot, \rightarrow, 1)$ is a hoop.

Corollary 3.35. Let $(E, \wedge, \sim, 1)$ be a good EQ-algebra that is not involutive and $x \odot y=x \wedge y$, for any $x, y \in E$. If $(x \odot y) \rightarrow z=x \rightarrow(y \rightarrow z)$, then $(E, \odot, \rightarrow, 1)$ is a hoop. 


\subsection{Relation with fuzzy implication algebras}

In this section, we try to investigate that under what condition hoops will be fuzzy implication algebras.

Definition 3.36. 23] A fuzzy implication algebra ( FI-algebra) is an algebra $(A, \rightarrow, 0)$ of type $(2,0)$ satisfying the following axioms, for any $x, y, z \in A$,

$(F I 1) x \rightarrow(y \rightarrow z)=y \rightarrow(x \rightarrow z)$,

(FI2) $x \rightarrow x=1$,

(FI3) $x \rightarrow y=y \rightarrow x=1$ implies $x=y$,

$(F I 4) \quad(x \rightarrow y) \rightarrow((y \rightarrow z) \rightarrow(x \rightarrow z))=1$,

$(F I 5) \quad 0 \rightarrow 1=1$.

Where $1=0 \rightarrow 0$.

An FI-algebra $A$ is called normal if $\left(x^{\prime}\right)^{\prime}=x$, for any $x \in A$, where $x^{\prime}=x \rightarrow 0$.

Lemma 3.37. Let $(A, \odot, \rightarrow, 0,1)$ be a bounded hoop and for any $x, y \in A, x \otimes y=\left(x \rightarrow y^{\prime}\right)^{\prime}$. If $A$ has $(D N P)$, then two products $\odot$ and $\otimes$ conside.

Proof. Let $(A, \odot, \rightarrow, 0,1)$ be a bounded hoop with (DNP) and for any $x, y \in A, x \otimes y=\left(x \rightarrow y^{\prime}\right)^{\prime}$. Then by (HP3) and (DNP),

$$
x \otimes y=\left(x \rightarrow y^{\prime}\right)^{\prime}=(x \rightarrow(y \rightarrow 0))^{\prime}=((x \odot y) \rightarrow 0)^{\prime}=(x \odot y)^{\prime \prime}=x \odot y .
$$

Theorem 3.38. BCK-algebras and FI-algebras coincide.

Proof. Let $(A, *, 0)$ be a BCK-algebra. Then it is enough to define $x * y=y \rightarrow x$, for all $x, y \in A$ and consider that the constant is 1 . Hence, $(A, \rightarrow, 0)$ is an FI-algebra.

Theorem 3.39. $(A, \odot, \rightarrow, 0,1)$ is a bounded hoop with $(D N P)$ if and only if $(A, \rightarrow, 0)$ is a normal FI-algebra with product $\otimes$ such that $(x \rightarrow y) \rightarrow y=(y \rightarrow x) \rightarrow x$, for any $x, y \in A$.

Proof. $(\Rightarrow)$ Let $(A, \odot, \rightarrow, 0,1)$ be a bounded hoop with (DNP). Then by (HP2) and (HP3), we have (FI1) and (FI2). Since $(A, \leq)$ is a poset, if $x \rightarrow y=y \rightarrow x=1$, for any $x, y \in A$, then $x=y$. Moreover, by Proposition 2.2(ix) and (vi), we have (FI4) and (FI5). Since $A$ has (DNP), $A$ is normal. Therefore, $A$ is a normal $F I$-algebra.

$(\Leftarrow)$ Let $(A, \rightarrow, 0)$ be a normal $F I$-algebra with product $\otimes$ such that for any $x, y \in A,(x \rightarrow$ $y) \rightarrow y=(y \rightarrow x) \rightarrow x$. Then by [24, Corollary 7.2.1], $A$ is an $M V$-algebras. Hence, by Theorem 3.13. $(A, \odot, \rightarrow, 0,1)$ is a bounded hoop. Moreover, since $A$ is normal, then $A$ has (DNP).

\subsection{Relation with Heyting (Hertz) algebras}

In this section, we investigate the relation among hoop and Heyting (Hertz) algebras.

Definition 3.40. [9] A Hertz algebra is an algebra $(A, \rightarrow, \wedge, 1)$ of type $(2,2,0)$ which, for all $x, y, z \in A$, it satisfies the following axioms:

(HT1) $x \rightarrow x=1$,

$(H T 2) \quad y \wedge(x \rightarrow y)=y$,

(HT3) $x \wedge(x \rightarrow y)=x \wedge y$,

(HT4) $x \rightarrow(y \wedge z)=(x \rightarrow y) \wedge(x \rightarrow z)$. 
Theorem 3.41. If $(A, \odot, \rightarrow, 1)$ is a hoop and $x \wedge(x \rightarrow y)=x \wedge y$, for any $x, y \in A$, then $(A, \rightarrow, \wedge, 1)$ is a Hertz algebra.

Proof. Let $(A, \odot, \rightarrow, 1)$ be a hoop such that $x \wedge(x \rightarrow y)=x \wedge y$, for any $x, y \in A$. Then by (HP2), we have (HT1). By Proposition 2.2(iv), $y \leq x \rightarrow y$, for all $x, y \in A$. Then $y \wedge(x \rightarrow y)=y$, and so we have (HT2). Moreover, by Proposition 2.2(xiv), we obtain (HT4). Hence, by assumption, we have (HT3). Therefore, $(A, \rightarrow, \wedge, 1)$ is a Hertz algebra.

Example 3.42. Let $A=\{0, a, b, 1\}$ be a set such that $0 \leq a \leq b \leq 1$. Define the operations $\rightarrow$ and $\odot$ on $A$ as follows:

\begin{tabular}{c|cccc}
$\rightarrow$ & 0 & $a$ & $b$ & 1 \\
\hline 0 & 1 & 1 & 1 & 1 \\
$a$ & 0 & 1 & 1 & 1 \\
$b$ & 0 & $a$ & 1 & 1 \\
1 & 0 & $a$ & $b$ & 1
\end{tabular}

\begin{tabular}{c|cccc}
$\odot$ & 0 & $a$ & $b$ & 1 \\
\hline 0 & 0 & 0 & 0 & 0 \\
$a$ & 0 & $a$ & $a$ & $a$ \\
$b$ & 0 & $a$ & $b$ & $b$ \\
1 & 0 & $a$ & $b$ & 1
\end{tabular}

Then $(A, \odot, \rightarrow, 0,1)$ is a bounded hoop. Also, it is easy to see that $x \wedge(x \rightarrow y)=x \wedge y$, for all $x, y \in A$. Then $A$ is a Hertz algebra.

Definition 3.43. [4] $A$ Heyting algebra is an algebra $(A, \vee, \wedge, \rightarrow, 1)$ of type $(2,2,2,0)$, where $(A, \vee, \wedge, 1)$ is a lattice with the greatest element and the binary operation $\rightarrow$ on $A$ verifies, for any $x, y, z \in A$, by

$$
x \leq y \rightarrow z \text { if and only if } x \wedge y \leq z .
$$

Theorem 3.44. Let $(A, \odot, \rightarrow, 1)$ be $a \vee$-hoop. Then $(A, \vee, \wedge, \rightarrow, 1)$ is a Heyting algebra if $x \leq$ $y \rightarrow z$, then $x \wedge y \leq z$, for any $x, y, z \in A$.

Proof. Let $(A, \odot, \rightarrow, 1)$ be a $\vee$-hoop. By Remark 2.6, $A$ is a distributive lattice with upper bounded. Let $x \wedge y \leq z$, for $x, y, z \in A$. By Proposition 2.2(iii), $x \odot y \leq x \wedge y$, and so $x \odot y \leq z$. Then by Proposition 2.2(ii), $x \leq y \rightarrow z$. Now, by assumption, if $x \leq y \rightarrow z$, then $x \wedge y \leq z$, for any $x, y, z \in A$. Hence, $(A, \wedge, \vee, \rightarrow, 1)$ is a Heyting algebra.

The following example shows that the condition in previous theorem is necessary.

Example 3.45. Let $A=\{0, a, b, c, d, 1\}$ be a poset with Hasse diagram in Figure 1, and Cayley tables as follows,

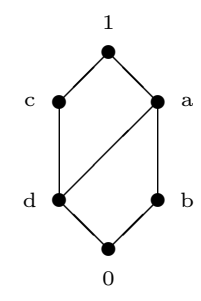

Figure 1: The Hasse diagram of $A$ 


\begin{tabular}{c|cccccc}
$\rightarrow$ & 1 & $a$ & $b$ & $c$ & $d$ & 0 \\
\hline 1 & 1 & $a$ & $b$ & $c$ & $d$ & 0 \\
$a$ & 1 & 1 & $a$ & $c$ & $c$ & $d$ \\
$b$ & 1 & 1 & 1 & $c$ & $c$ & $c$ \\
$c$ & 1 & $a$ & $b$ & 1 & $a$ & $b$ \\
$d$ & 1 & 1 & $a$ & 1 & 1 & $a$ \\
0 & 1 & 1 & 1 & 1 & 1 & 1
\end{tabular}

\begin{tabular}{l|llllll}
$\odot$ & 1 & $a$ & $b$ & $c$ & $d$ & 0 \\
\hline 1 & 1 & $a$ & $b$ & $c$ & $d$ & 0 \\
$a$ & $a$ & $b$ & $b$ & $d$ & 0 & 0 \\
$b$ & $b$ & $b$ & $b$ & 0 & 0 & 0 \\
$c$ & $c$ & $d$ & 0 & $c$ & $d$ & 0 \\
$d$ & $d$ & 0 & 0 & $d$ & 0 & 0 \\
0 & 0 & 0 & 0 & 0 & 0 & 0
\end{tabular}

For any $x, y \in A$, we define, $x \wedge y=x \odot(x \rightarrow y)$ and $x \vee y=((x \rightarrow y) \rightarrow y) \wedge((y \rightarrow x) \rightarrow x)$. Then $(A, \odot, \rightarrow, 0,1)$ is a bounded $\vee$-hoop. We claim that $A$ is not a Heyting algebra. Since $d \leq a \rightarrow b$, but $d \wedge a=d \not \leq b$, because, $b$ and $d$ are incomparable. So, $A$ is not a Heyting algebra.

\subsection{Relation with $R_{0}$-algebras (lattice implication algebras, Hilbert algebras)}

In this section, we investigate the relation among hoops and lattice implication algebras, $R_{0^{-}}$ algebras and Hilbert algebras.

Definition 3.46. 24] Let $(A, \vee, \wedge, 0,1)$ be a bounded lattice with an order-reversing involution', 1 and 0 be the greatest and the smallest element of $A$, respectively. Then the algebraic structure $\left(A, \vee, \wedge, \rightarrow,{ }^{\prime}, 0,1\right)$ of type $(2,2,2,1,0,0)$ is called a lattice implication algebra if the following conditions hold, for any $x, y, z \in A$ :

(I1) $x \rightarrow(y \rightarrow z)=y \rightarrow(x \rightarrow z)$;

(I2) $\quad x \rightarrow x=1$;

(I3) $x \rightarrow y=y^{\prime} \rightarrow x^{\prime}$;

(I4) $x \rightarrow y=y \rightarrow x=1$ implies $x=y$;

(I5) $\quad(x \rightarrow y) \rightarrow y=(y \rightarrow x) \rightarrow x$;

(I6) $\quad(x \vee y) \rightarrow z=(x \rightarrow z) \wedge(y \rightarrow z)$;

$($ I7) $\quad(x \wedge y) \rightarrow z=(x \rightarrow z) \vee(y \rightarrow z)$.

Theorem 3.47. ( $i)$ If $(A, \wedge, \vee, \rightarrow, 0,1)$ is a lattice implication algebra with a binary operation $\otimes$, then $(A, \otimes, \rightarrow, 0,1)$ is a bounded hoop.

(ii) If $(A, \odot, \rightarrow, 0,1)$ is a bounded $\vee$-hoop with (DNP) and condition (M1), then $(A, \wedge, \vee, \rightarrow, 0,1)$ is a lattice implication algebra.

Proof. $(i)$ Let $(A, \wedge, \vee, \rightarrow, 0,1)$ be a lattice implication algebra with a binary operation $\otimes$. By Lemma 3.37 and [24, Corollary 2.1.3], $(A, \wedge, \vee, \otimes, \rightarrow, 1)$ is a residuated lattice. Since $A$ has (DNP), by (I5) and Lemma 3.20 , for any $x, y \in A$, we have,

$$
x \otimes(x \rightarrow y)=\left(x \rightarrow(x \rightarrow y)^{\prime}\right)^{\prime}=\left(\left(y^{\prime} \rightarrow x^{\prime}\right) \rightarrow x^{\prime}\right)^{\prime}=\left(x^{\prime} \vee y^{\prime}\right)^{\prime}=x \wedge y .
$$

Hence, $x \otimes(x \rightarrow y)=x \wedge y$. Then by Theorem 3.5, $(A, \otimes, \rightarrow, 0,1)$ is a bounded hoop.

(ii) Let $(A, \odot, \rightarrow, 0,1)$ be a bounded $\vee$-hoop with (DNP) and condition (M1). By Theorem 3.5, $(A, \wedge, \vee, \odot, \rightarrow, 0,1)$ is a residuated lattice, by Proposition 3.2 and (M1), (I6) and (I7) hold. Also, by (HP2) and (HP3), (I1) and (I2) hold. Let $x \rightarrow y=y \rightarrow x=1$, for some $x, y \in A$. Then $x \leq y$ and $y \leq x$. Since $A$ is a poset, we get that $x=y$. Moreover, since $A$ has (DNP), by Lemma 3.20 , (I3) and (I5) hold. Therefore, $(A, \wedge, \vee, \rightarrow, 0,1)$ is a lattice implication algebra. 
Definition 3.48. 21] Let $(A, \vee, \wedge, 0,1)$ be a bounded distributive lattice with two operations' and $\rightarrow$. Then $A$ is called an $R_{0}$-algebra if, for any $x, y, z \in A$, the following conditions hold:

$(R 1) \quad x \rightarrow y=y^{\prime} \rightarrow x^{\prime}$;

(R2) $1 \rightarrow x=x$;

(R3) $y \rightarrow z \leq(x \rightarrow y) \rightarrow(x \rightarrow z)$;

(R4) $x \rightarrow(y \rightarrow z)=y \rightarrow(x \rightarrow z)$;

$(R 5) \quad x \rightarrow(y \vee z)=(x \rightarrow y) \vee(x \rightarrow z)$;

$(R 6) \quad(x \rightarrow y) \vee\left((x \rightarrow y) \rightarrow\left(x^{\prime} \vee y\right)\right)=1$;

(R7) $x \rightarrow x=1$;

$(R 8) x \rightarrow(y \wedge z)=(x \rightarrow y) \wedge(x \rightarrow z)$.

Theorem 3.49. [24, Corollary 7.2.2] (1) If $(M, \vee, \wedge, 0,1)$ is an $R_{0}$-algebra such that for any $x, y \in M,(x \rightarrow y) \rightarrow y=(y \rightarrow x) \rightarrow x$, then $M$ is a lattice implication algebra.

(2) If $\left(L, \wedge, \vee, \rightarrow,{ }^{\prime}, 0,1\right)$ is a lattice implication algebra such that for any $x, y \in L,(x \rightarrow y) \vee((x \rightarrow$ $\left.y) \rightarrow\left(x^{\prime} \vee y\right)\right)=1$, then $L$ is an $R_{0}$-algebra.

Theorem 3.50. (i) If $(A, \vee, \wedge, \rightarrow, 0,1)$ is an $R_{0}$-algebra such that $(x \rightarrow y) \rightarrow y=(y \rightarrow x) \rightarrow x$, for any $x, y \in A$, then $(A, \odot, \rightarrow, 0,1)$ is a bounded hoop.

(ii) If $(A, \odot, \rightarrow, 0,1)$ is a bounded $\vee$-hoop with (DNP) satisfying in conditions (M1) and $\left(R_{6}\right)$, then $(A, \vee, \wedge, \rightarrow, 0,1)$ is an $R_{0}$-algebra.

Proof. $(i)$ Let $(A, \vee, \wedge, \rightarrow, 0,1)$ be an $R_{0}$-algebra and for any $x, y \in A, x \otimes y=\left(x \rightarrow y^{\prime}\right)^{\prime}$. Then by Lemma 3.37, $\otimes$ and $\odot$ are equal. If for any $x, y \in A(x \rightarrow y) \rightarrow y=(y \rightarrow x) \rightarrow x$, then by Theorem 3.49. $\left(A, \vee, \wedge, \rightarrow,^{\prime}, 0,1\right)$ is a lattice implication algebra. Thus, by Theorem 3.47, $(A, \odot, \rightarrow, 0,1)$ is a bounded hoop.

(ii) Let $(A, \odot, \rightarrow, 0,1)$ be a bounded $\vee$-hoop with (DNP) satisfying in conditions (M1). Then by Theorem 3.47, $\left(A, \vee, \wedge, \rightarrow{ }^{\prime}, 0,1\right)$ is a lattice implication algebra, and if $\left(R_{6}\right)$ holds, then by Corollary 3.49 , $(A, \vee, \wedge, \rightarrow, 0,1)$ is an $R_{0}$-algebra.

Let us remark that $R_{0^{-}}$-algebras and hoops are incomparable. Indeed, not all hoops are $R_{0^{-}}$ algebras. It is noticeable that, hoop is a meet semilattice with respect to the meet operator $a \wedge b=a \odot(a \rightarrow b)$ but it has not a lattice structure. The following example indicates that any $R_{0}$-algebra is not a hoop, in general, and conversely. It shows that the conditions in Theorem 3.50 are necessary.

Example 3.51. ( $i)$ Let $(A, \odot, \rightarrow, 1)$ be the hoop as in Example 3.22. It is clear that $A$ does not have (DNP) and $b=a \rightarrow b \neq a^{\prime} \rightarrow b^{\prime}=1$, so (R1) does not hold. Hence, $A$ is not an $R_{0}$-algebra. (ii) Let $A=\{0, a, b, c, 1\}$ be a set such that $0 \leq a \leq b \leq 1$ and define the operation $\rightarrow$ on $A$ as follows:

\begin{tabular}{c|ccccc}
$\rightarrow$ & 0 & $a$ & $b$ & $c$ & 1 \\
\hline 0 & 1 & 1 & 1 & 1 & 1 \\
$a$ & $c$ & 1 & 1 & 1 & 1 \\
$b$ & $b$ & $b$ & 1 & 1 & 1 \\
$c$ & $a$ & $a$ & $b$ & 1 & 1 \\
1 & 0 & $a$ & $b$ & $c$ & 1
\end{tabular}

Let $\neg a=c, \neg b=b$ and $\neg c=a$. Then $(A, \wedge, \vee, \neg, \rightarrow, 0,1)$ is an $R_{0}$-algebra, where $x \wedge y=\min \{x, y\}$ and $x \vee y=\max \{x, y\}$. By routine calculation, we can see that

$$
c=(a \rightarrow c) \rightarrow c \neq(c \rightarrow a) \rightarrow a=a \rightarrow a=1 .
$$


Let define $x \odot y=\neg(x \rightarrow \neg y)$, for any $x, y \in A$. We claim that $(A, \odot, \rightarrow, 0,1)$ is not a hoop, because

$$
c \odot(c \rightarrow a)=c \odot a=\neg(c \rightarrow \neg a)=\neg(c \rightarrow c)=0 \neq c \wedge a=a
$$

Definition 3.52. [10] A Hilbert algebra is a tripe $(A, \rightarrow, 1)$ of type $(2,0)$ such that the following three axioms are satisfied, for all $x, y, z \in A$ :

$(H 1) x \rightarrow(y \rightarrow x)=1$,

$(H 2) \quad x \rightarrow(y \rightarrow z)=(x \rightarrow y) \rightarrow(x \rightarrow z)$,

(H3) if $x \rightarrow y=y \rightarrow x=1$, then $x=y$.

The Hilbert algebra induces a partial order $\leq$ on $A$, defined by, $x \leq y$ if and only if $x \rightarrow y=1$ and 1 is the greatest element of the induced poset $(A, \leq)$. A Hilbert algebra $A$ is called bounded if there exists $0 \in A$ such that $0 \leq x$, for any $x \in A$.

Theorem 3.53. ( $i)$ If $(A, \odot, \rightarrow, 0,1)$ is a bounded hoop with (DNP) such that $x^{2}=x$, for any $x \in A$, then $(A, \rightarrow, 0,1)$ is a bounded Hilbert algebra.

(ii) If $(A, \rightarrow, 0,1)$ is a bounded Hilbert algebra with (DNP), then $(A, \odot, \rightarrow, 0,1)$ is a bounded hoop.

Proof. $(i)$ Let $(A, \odot, \rightarrow, 0,1)$ be a bounded hoop with (DNP) such that $x^{2}=x$, for any $x \in A$. Then by Proposition 2.2(iv), $x \leq y \rightarrow x$. Thus, $x \rightarrow(y \rightarrow x)=1$, and so (H1) holds. Moreover, since $(A, \leq)$ is a poset, if $x \rightarrow y=y \rightarrow x=1$, then $x \leq y$ and $y \leq x$. Hence, $x=y$. So, we have (H3). Now, it is enough to prove (H2). By Proposition 2.2(iv), $y \leq x \rightarrow y$, for any $x, y \in A$ and by Proposition 2.2(xiii) and (xii),

$$
x \rightarrow((x \rightarrow y) \rightarrow z) \leq x \rightarrow(y \rightarrow z)
$$

Then by (HP3),

$$
(x \rightarrow y) \rightarrow(x \rightarrow z) \leq x \rightarrow(y \rightarrow z)
$$

Conversely, by (HP3), for all $x, y, z \in A$,

$$
[x \rightarrow(y \rightarrow z)] \rightarrow[(x \rightarrow y) \rightarrow(x \rightarrow z)]=[(x \rightarrow y) \odot x \odot(x \rightarrow(y \rightarrow z))] \rightarrow z
$$

Since $x \rightarrow(y \rightarrow z) \leq x \rightarrow(y \rightarrow z)$, by Proposition 2.2(ii),

$$
x \odot(x \rightarrow(y \rightarrow z)) \leq y \rightarrow z
$$

Then by Proposition 2.2(xi) and (x),

$$
(x \rightarrow y) \odot x \odot(x \rightarrow(y \rightarrow z)) \leq(x \rightarrow y) \odot(y \rightarrow z) \leq x \rightarrow z
$$

Thus,

$$
x^{2} \odot(x \rightarrow y) \odot(x \rightarrow(y \rightarrow z)) \leq z
$$

Since $x^{2}=x$, we have

$$
x \odot(x \rightarrow y) \odot(x \rightarrow(y \rightarrow z)) \leq z
$$

Hence, by (HP3),

$$
x \rightarrow(y \rightarrow z) \leq(x \rightarrow y) \rightarrow(x \rightarrow z)
$$

Therefore, $(A, \rightarrow, 0,1)$ is a bounded Hilbert algebra.

(ii) Let $(A, \rightarrow, 0,1)$ be a bounded Hilbert algebra with (DNP) and $x \otimes y=\left(x \rightarrow y^{\prime}\right)^{\prime}$, for any $x, y \in A$. Then, by [9, Theorem 1.3.1], $A$ is a Boolean algebra. Moreover, by [24, Example 2.1.1], any Boolean algebra $A$ with $x \rightarrow y=x^{\prime} \vee y$, for any $x, y \in A$, is an $M V$-algebra. Therefore, by Theorem 3.13, $(A, \odot, \rightarrow, 0,1)$ is a bounded hoop. 
The following example shows that every hoop is not a Hilbert algebra, in general.

Example 3.54. If $(A, \odot, \rightarrow, 1)$ is the hoop as in Example 3.45 , then $a \odot a=b$, and so $x^{2} \neq x$, for any $x \in A$. Now, since

$$
a \rightarrow(d \rightarrow b)=a \rightarrow a=1 \neq a=c \rightarrow a=(a \rightarrow d) \rightarrow(a \rightarrow b) .
$$

Hence, $(A, \rightarrow, 0,1)$ is not a bounded Hilbert algebra.

In the following figure, we have the relation among hoop and some of other logical algebras.

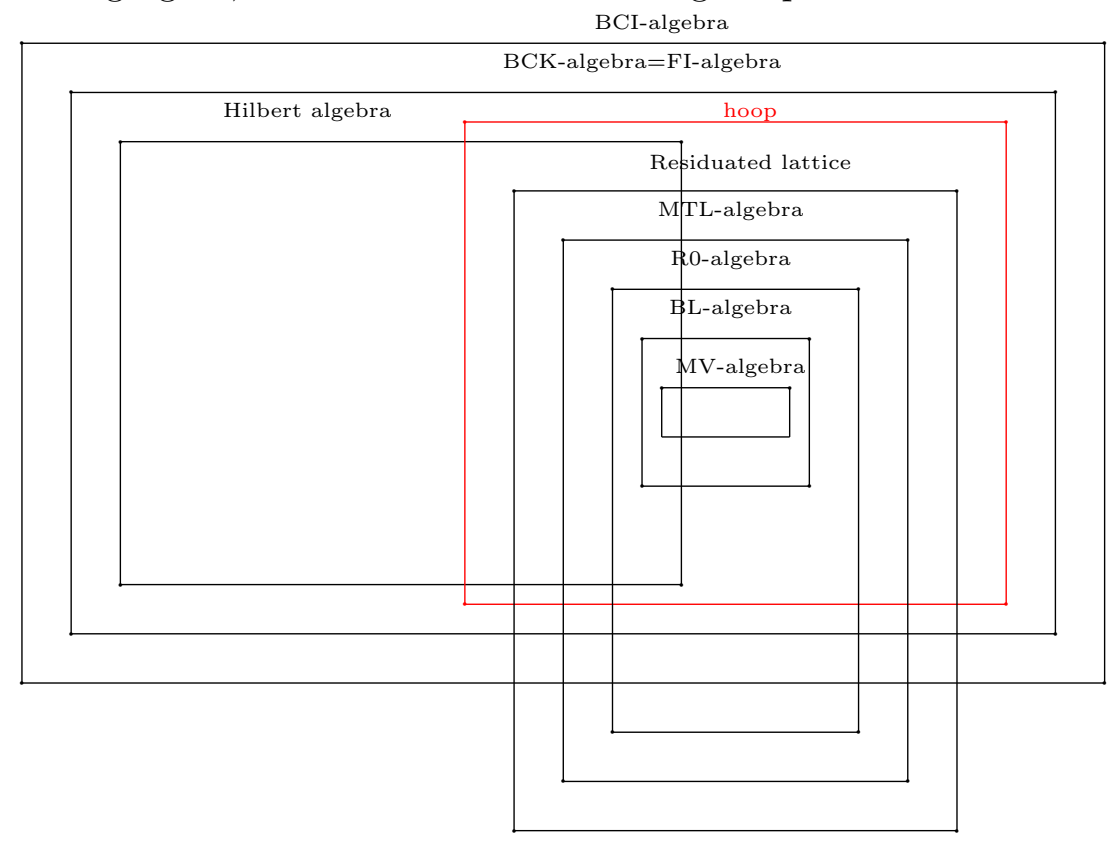

Figure 2: Relation among hoop and some of logical algebras

\section{Conclusions}

The aim of this paper is to study the relation among hoops and other logical algebras such as residuated lattice, Hilbert and $M T L$-algebras, etc. In this way, we can see that under what conditions hoops are equivalent to these logical algebras. So, many results that are proved for these logical algebras, hold for hoops.

\section{References}

[1] P. Aglianò., I.M. Ferreirim, F. Montagna, Basic hoops: An algebraic study of continuous t-norm, Studia Logica, 87(1) (2007), 73-98.

[2] S.Z. Alavi, R.A. Borzooei, M. Aaly Kologani, Filter theory of pseudo hoop-algebras, Italian Journal of Pure and Applied Mathematics, 37 (2017), 619-632.

[3] W.J. Blok, I.M.A. Ferreirim, On the structure of hoops, Algebra Universalis, 43(2-3) (2000), 233-257. 
[4] V. Boicescu, A. Filipoiu, G. Georgescu, S. Rudeanu, Lukasiewicz-Moisil algebras, Annals of Discrete Mathematics, 49 (1991).

[5] R.A. Borzooei, M. Aaly Kologani, Local and perfect semihoops, Journal of Intelligent and Fuzzy Systems, 29(1) (2015), 223-234.

[6] R.A. Borzooei, M. Aaly Kologani, State hoops, Mathematica Slovaca, 67(1) (2017), 1-16.

[7] B. Bosbach, Komplementäre Halbgruppen. Axiomatik und Arithmetik, Fundamenta Mathematicae, 64 (1969), 257-287.

[8] B. Bosbach, Komplementäre Halbgruppen. Kongruenzen und Quotienten, Fundamenta Mathematicae, 69 (1970), 1-14.

[9] D. Busneag, Hertz algebras of fractions and maximal Hertz algebra of quotients, Mathematica Japonica, 39(3) (1993), 461-469.

[10] D. Busneag, A note on deductive systems of a Hilbert algebra, Kobe Journal of Mathematics, 2 (1985), 29-35.

[11] C.C. Chang, Algebraic analysis of many valued logics, Transactions of the American Mathematical Society, 88(2) (1958), 467-490.

[12] L.Ciungu, On pseudo-BCK-algebras with pseudo double negation, Annals of the University of Craiova, Mathematics and Computer Science Series, 37(1) (2010), 19-26.

[13] M. El-Zekey, V.N. Ak, R. Mesiar, On good EQ-algebras, Fuzzy Sets and Systems, 178 (2011), $1-23$.

[14] F. Esteva, L. Godo, Monoidal t-norm based logic: Towards a logic for left-continuous t-norms, Fuzzy Sets and Systems, 124(3) (2001), 271-288.

[15] G. Georgescu, L. Leustean, V. Preoteasa, Pseudo-hoops, Journal of Multiple-Valued Logic and Soft Computing, 11(1-2) (2005), 153-184.

[16] P. Hájek, Metamathematics of fuzzy logic, Springer Science and Business Media, 4 (1998).

[17] A. Iorgulescu, Algebras of logic as BCK-algebras, Bucharest: Editura ASE, (2008).

[18] K. Iseki, Y. Imai, On axiom systems of propositional calculi, XIV Proc. Japan Academy, 42(1) (1966), 19-22.

[19] S. Jenei, Equality algebras, Studia Logica, 100 (2012), 1201-1209.

[20] D.W. Pei, A unified normal residuated based logic system and its completeness, Southeast Asian Bulletin of Mathematics, 28(6) (2004), 1089-1098.

[21] G.J. Wang, Non-classical mathematical logic and approximate reasoning, Beijing: Science Press (2000).

[22] M. Ward, R.P. Dilworth, Residuated lattices, Transactions of the American Mathematical Society, 45(3) (1939), 335-354.

[23] W.M. Wu, Fuzzy implication algebra, Fuzzy Systems and Mathematics, 4(1) (1990), 56-64. 
[24] Y. Xu, D. Ruan, K. Qin, J. Liu, Lattice valued logic, Studies in Fuzziness and Soft Computing, 132 (2003).

[25] F. Zebardast, R.A. Borzooei, M. Aaly Kologani, Results on equality algebras, Information Sciences, 381 (2017), 270-282. 